\title{
Abdominal Cerebrospinal Fluid Pseudocysts in Children: A Complication of Ventriculoperitoneal Shunt
}

\author{
Harshil C. Shah ${ }^{1}$ Divik H. Mittal ${ }^{1}$ Jaimin K. Shah ${ }^{1}$ \\ ${ }^{1}$ Department of Neurosurgery, B. J. Medical College and Civil \\ Hospital, Ahmedabad, Gujarat, India \\ Address for correspondence Divik H. Mittal, MS, 2, Vithal Nagar, Civil \\ Camp Road, Shahibaug, Ahmedabad 380004, Gujarat, India \\ (e-mail: drdivik@yahoo.com). \\ Indian J Neurosurg 2015;4:49-51.
}

\begin{abstract}
Keywords

- abdominal cerebrospinal fluid pseudocyst

- ventriculoperitoneal shunt

- ventriculoatrial shunt

Abdominal cerebrospinal fluid (CSF) pseudocyst is a rare complication of ventriculoperitoneal (VP) shunt. It is characterized by collection of cerebrospinal fluid in the peritoneal cavity, containing the distal end of the VP shunt catheter and is surrounded by a wall composed of fibrous tissue without an epithelial lining. In this article, we report four cases of abdominal cerebrospinal fluid pseudocyst in children with VP shunt, performed for tuberculous meningitis with hydrocephalus. We have described the etiopathogenesis, the symptomatology, the diagnostic investigations, the treatment method adopted, and proposed a simple and effective treatment protocol that has resulted in rapid resolution of symptoms in all our patients. Also, one of our cases had the largest abdominal CSF pseudocyst reported in the literature with respect to weight and height of the child.
\end{abstract}

\section{Introduction}

Abdominal cerebrospinal fluid (CSF) pseudocyst is an uncommon, but an important complication of ventriculoperitoneal (VP) shunt, with its incidence ranging from 0.33 to $6.8 \%{ }^{1-4}$ Abdominal CSF pseudocyst usually occurs in children ${ }^{1,3,5}$ and is extremely rare in adults. ${ }^{5}$ We report four cases of abdominal CSF pseudocyst diagnosed and treated at our institute according to our set protocols. To the best of our knowledge, one of our cases, a 2-year-old male child had the largest abdominal CSF pseudocyst reported in the literature with respect to weight and height of the child.

\section{Case History}

We treated four patients of abdominal CSF pseudocyst with VP shunts. Their ages ranged from 2 to 16 years. VP shunt was done in all the patients for treatment of tuberculous meningitis with hydrocephalus. Their ages at the time the shunt was performed ranged from 6 months to 10 years. The time interval from shunt surgery to appearance of symptoms ranged from 1 to 13 years. The patients presented with abdominal pain and either a lump in the abdomen or abdominal distension. One of these patients, a 2-year-old male child also presented with altered sensorium and vomiting, suggesting shunt malfunction. His weight and height were $9 \mathrm{~kg}$ and $78 \mathrm{~cm}$, respectively. Abdominal ultrasonography (USG) showed a cystic lesion in peritoneal cavity with shunt tube seen within the cyst. The diagnosis was confirmed on abdominal computed tomography (CT) scan, which showed a large, well-defined, homogenous, lowdensity lesion in intraperitoneal compartment with shunt tip seen within the lesion (-Fig. 1A, B). CT brain was also done in all patients and one patient had findings suggestive of moderate hydrocephalus.

All patients underwent emergency laparotomy and cyst was drained, cyst wall biopsy taken, and the shunt was externalized. CSF studies showed no abnormal findings, but two patients had positive CSF culture reports ( - Table $\mathbf{1})$. They received prophylactic antibiotics initially and subsequently according to the culture and sensitivity reports. Ventriculoatrial shunt was done in the next stage, once the infection was ruled out or controlled. published online March 20, 2015
DOI http://dx.doi.org/ 10.1055/s-0035-1549059. ISSN 2277-954X. (c) 2015 Neurological Surgeons' Society of India

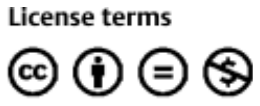



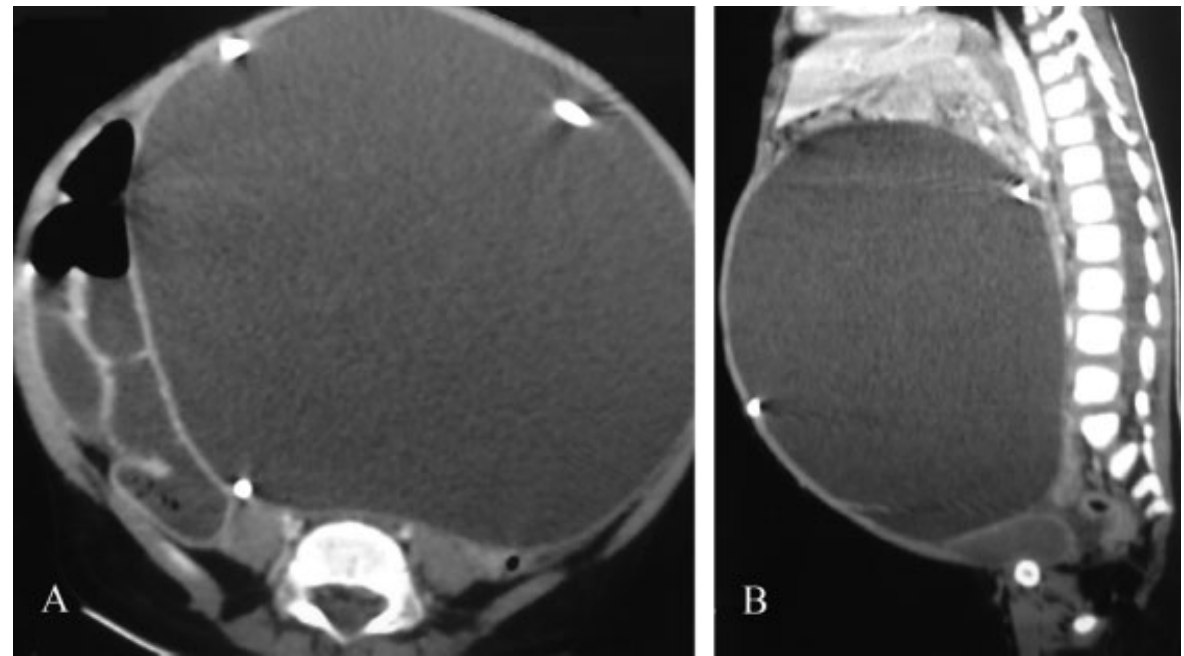

Fig. 1 Abdominal CT scan of case 1 (A: axial, B: sagittal) showing a large, well-defined, homogenous, low-density lesion, measuring $12 \times 15 \times 14 \mathrm{~cm}$ and extending from epigastrium up to pelvis. Ventriculoperitoneal (VP) shunt tip is seen within the lesion.

Histopathology reports showed cyst wall-like structure with fibrocollagenous vascularized connective tissue without epithelial lining. There were mix inflammatory infiltrates suggestive of nonspecific inflammatory changes. There was no evidence of tuberculosis. All patients had an uneventful recovery and were discharged in good clinical condition. A routine $\mathrm{X}$-ray chest and $2 \mathrm{D}$ echo was done to confirm the position of ventriculoatrial shunt's tip. All were asymptomatic on their routine follow-up and USG showed no intra-abdominal fluid collection.

\section{Discussion}

Abdominal CSF pseudocyst is characterized by collection of CSF in the peritoneal cavity, containing the distal end of the VP shunt and is surrounded by a wall composed of fibrous tissue without an epithelial lining. ${ }^{4}$ The underlying mechanisms involved in the formation of abdominal CSF pseudocyst are still unknown; however, inflammatory process, either sterile or infectious, is usually regarded as the main causative factor. ${ }^{1,2,4}$ It could be due to focal peritonitis around the distal end of the shunt catheter. The inflammatory response could be sterile due to some component of the shunt tube or due to the high concentration of proteins in the CSF draining into the peritoneal cavity. ${ }^{6}$ Previous abdominal surgery could be responsible for the formation of peritoneal adhesions, thus preventing absorption of CSF. Two of our patients had positive culture reports suggesting local infection, which could have been the reason behind the cyst formation. Although the reports were negative in the other two patients, it is possible that previous infection could have resulted in peritoneal adhesions, thus decreasing CSF absorption.

Table 1 Details of four children with abdominal CSF pseudocyst

\begin{tabular}{|l|l|l|l|l|l|}
\hline Patient & Age $(\mathrm{y})$ & $\begin{array}{l}\text { Age when shunt } \\
\text { was performed }\end{array}$ & Sex & CSF culture & CT abdomen/CT brain \\
\hline 1 & 2 & $6 \mathrm{mo}$ & $\mathrm{M}$ & Klebsiella pneumoniae & $\begin{array}{l}12 \times 15 \times 14 \mathrm{~cm} \text { well-defined lesion } \\
\text { extending from epigastrium up to pelvis, with } \\
\text { shunt tip seen. } \\
\text { Moderate communicating hydrocephalus }\end{array}$ \\
\hline 2 & 16 & $3 \mathrm{y}$ & $\mathrm{M}$ & Staphylococcus aureus & $\begin{array}{l}9 \times 16 \times 12 \mathrm{~cm} \text { loculated collection } \\
\text { extending from epigastrium to umbilicus, with } \\
\text { shunt tip seen }\end{array}$ \\
\hline 3 & 15 & $10 \mathrm{y}$ & $\mathrm{F}$ & - & $\begin{array}{l}8 \times 10 \times 7 \mathrm{~cm} \text { well-defined lesion extending } \\
\text { from umbilicus up to pelvis, with shunt tip } \\
\text { seen }\end{array}$ \\
\hline 4 & 5 & $4 \mathrm{y}$ & $\mathrm{M}$ & - & $\begin{array}{l}8 \times 12 \times 9 \mathrm{~cm} \text { well-defined lesion in } \\
\text { abdomen, with shunt tip seen within the lesion }\end{array}$ \\
\hline
\end{tabular}

Abbreviations: CSF, cerebrospinal fluid; $\mathrm{CT}$, computed tomography. 
Patients may present with abdominal pain, abdominal distension, abdominal lump, or symptoms of elevated intracranial pressure such as headache, vomiting, and altered sensorium, suggestive of shunt malfunction. It may present as an acute abdomen and therefore should be included in the differential diagnosis of acute abdomen. ${ }^{7,8}$

Plain radiographs are useful to rule out other causes of acute abdomen and help determine the continuity of the shunt. USG is best for fast and reliable diagnosis of abdominal CSF pseudocysts. ${ }^{4}$ However, a catheter adherent to a cyst wall is difficult to visualize with USG. CT is effective in the diagnosis of abdominal CSF pseudocysts, especially when they are large and deform the normal architecture of the abdomen. It can demonstrate both free floating catheters and those adherent to cyst walls. ${ }^{3,9}$

There are no fixed guidelines for treatment of abdominal CSF pseudocysts, and therefore, many treatment protocols have been described in the previous reports. ${ }^{2,4}$ Surgical treatment options include laparotomy with excision or biopsy of the cyst walls, CT- or USG-guided aspiration of the pseudocyst, and laparoscope-assisted drainage of the pseudocyst ${ }^{10}$-along with repositioning the distal peritoneal end of shunt in a different abdominal quadrant, shunt removal, external ventricular drainage, and conversion to either a ventriculoatrial or a ventriculopleural shunt. From our previous experiences with such patients, we have seen that repositioning the shunt in a different abdominal quadrant may result in recurrence of pseudocyst or shunt malfunction. CT, USG, or laparoscope-assisted drainage of the pseudocyst also results in recurrence of symptoms because of the continuous drainage of CSF into the abdominal cavity. Therefore unless the CSF drainage is diverted, pseudocyst or hydrocephalus due to shunt malfunction is liable to develop. According to our protocols, once the diagnosis is made, urgent laparotomy is done with drainage of cyst, cyst wall biopsy is taken, and shunt is externalized. Systemic antibacterial therapy is administered throughout the course of treatment. Once the infection is controlled or ruled out by CSF reports, ventriculoatrial shunt is done in the next stage.

In conclusion, early diagnosis and treatment can result in good outcome. Cyst drainage with externalization of shunt followed by ventriculoatrial shunt is an effective treatment, resulting in complete resolution of symptoms.

\section{Key Points}

1. Cyst drainage with externalization of shunt followed by ventriculoatrial shunt is an effective treatment of abdominal cerebrospinal fluid pseudocyst, resulting in complete resolution of symptoms.

2. Largest abdominal CSF pseudocyst reported in the literature.

\section{Source(s) of Support}

Nil.

\section{Acknowledgment}

We thank Dr. Jayesh A. Shelat (Professor and Head, Department of Neurosurgery, B.J. Medical College and Civil Hospital, Ahmedabad, Gujrat) for his valuable support and instructions on this article.

\section{References}

1 Rainov N, Schobess A, Heidecke V, Burkert W. Abdominal CSF pseudocysts in patients with ventriculo-peritoneal shunts. Report of fourteen cases and review of the literature. Acta Neurochir (Wien) 1994;127(1-2):73-78

2 Salomão JF, Leibinger RD. Abdominal pseudocysts complicating CSF shunting in infants and children. Report of 18 cases. Pediatr Neurosurg 1999;31(5):274-278

3 Pernas JC, Catala J. Case 72: pseudocyst around ventriculoperitoneal shunt. Radiology 2004;232(1):239-243

4 Mobley LW III, Doran SE, Hellbusch LC. Abdominal pseudocyst: predisposing factors and treatment algorithm. Pediatr Neurosurg 2005;41(2):77-83

5 Aparici-Robles F, Molina-Fabrega R. Abdominal cerebrospinal fluid pseudocyst: a complication of ventriculoperitoneal shunts in adults. J Med Imaging Radiat Oncol 2008;52(1):40-43

6 Erşahin Y, Mutluer S, Tekeli G. Abdominal cerebrospinal fluid pseudocysts. Childs Nerv Syst 1996;12(12):755-758

7 Grosfeld JL, Cooney DR, Smith J, Campbell RL. Intra-abdominal complications following ventriculoperitoneal shunt procedures. Pediatrics 1974;54(6):791-796

8 Hashimoto M, Yokota A, Urasaki E, Tsujigami S, Shimono M. A case of abdominal CSF pseudocyst associated with silicone allergy. Childs Nerv Syst 2004;20(10):761-764

9 Lee TG, Parsons PM. Ultrasound diagnosis of cerebrospinal fluid abdominal cyst. Radiology 1978;127(1):220

10 Popa F, Grigorean VT, Onose G, Popescu M, Strambu V, Sandu AM. Laparoscopic treatment of abdominal complications following ventriculoperitoneal shunt. J Med Life 2009;2(4):426-436 